\title{
TIEMPO Y CAPITAL FINANCIERO EN EL CAPITAL DE MARX*
}

Boris Salazar

"DOI: https://doi.org/10.18601/01245996.v20n39.05. E1 autor agradece la lectura de María del Pilar Castillo, las observaciones de Omar Díaz a una presentación oral y las observaciones críticas de dos evaluadores anónimos. Recepción: 23-11-2017, modificación final: 10-04-2018, aceptación: 11-052018. Sugerencia de citación: Salazar, B. (2018). Tiempo y capital financiero en El Capital de Marx. Revista de Economía Institucional, 20(39), 93-118.

a Magíster en Economía. Profesor, Departamento de Economía, Universidad del Valle, Cali, Colombia, [boris.salazar@correounivalle]. 


\section{Tiempo y capital financiero en El Capital de Marx}

Resumen.Este artículo sugiere que la metamorfosis de las deudas en derivados y títulos financieros, y de estos en deudas, genera redes de activos y obligaciones, que inducen la emergencia de distintos tiempos del capital, articulados en un sistema complejo, cuya dinámica hace posible sincronizarlos en forma de crisis de carácter global. La crisis se trata como un cambio de estado en un sistema complejo, precipitada por la conectividad generada por el apalancamiento creciente. Es un desarrollo de las teorías de la crisis y de la socialización de la deuda planteadas por Marx en El Capital.

Palabras clave: deuda, títulos financieros, red financiera, crisis global, Marx, tiempo absoluto, tiempo relacional; JEL: D85, E11, E44, G01, G15.

\section{Time and financial capital in Marx's Capital}

Abstract. This article argues that the metamorphosis of debts into financial derivatives and securities, and of the latter back into debt, generates networks of assets and liabilities inducing the emergence of different times for capital, articulated into a complex system whose dynamics make possible the synchronization of those times in the form of global crisis. The crisis is treated as a change of state in a complex system, precipitated by the connectivity generated by increasing leverage. It is a development of the theories of the crisis and of socialization of the debt raised by Marx in Capital.

Keywords: Debt, financial titles, financial network, global crisis, Marx, absolute time, relational time; JEL: D85, E11, E44, G01, G15.

\section{Tempo e capital financeiro em $O$ capital de Marx}

Resumo. Este artigo sugere que a metamorfose das dívidas em derivativos e títulos financeiros e destes em dívidas gera redes de ativos e obrigações, que induzem à emergência de diferentes tempos do capital, articulados num sistema complexo, cuja dinâmica torna possível sincronizá-los em forma de crise de caráter global. A crise é tratada como uma mudança de estado num sistema complexo, precipitada pela conectividade gerada pela alavancagem crescente. É um desenvolvimento das teorias da crise e da socialização da dívida propostas por Marx em O capital.

Palavras-chaves: dívida, títulos financeiros, rede financeira, crise global, Marx, tempo absoluto, tempo relacional; JEL: D85, E11, E44, G01, G15. 
$\mathrm{D}$ efenderé una hipótesis atrevida: la crisis financiera global de 2008 fue el resultado de la metamorfosis de la deuda (crédito) en nuevos títulos y derivados financieros que se transformaron en deudas que devinieron nuevos activos financieros, conformando una red interdependiente de activos y obligaciones, en la que el no pago local se convirtió - vía una conectividad creciente y la sincronización de los distintos tiempos del capital- en una ruptura generalizada del intercambio, es decir, en una crisis financiera global.

La metamorfosis de la deuda en nuevos títulos y derivados financieros, y de estos en deuda, solo fue posible por la funcionalidad de la forma monetaria del valor (Marx, 1976). Todas las metamorfosis involucradas implicaban la conversión esperada de la deuda y de los nuevos derivados financieros en dinero: la forma monetaria del valor. $\mathrm{Y}$ todas las mutaciones ocurridas solo fueron posibles porque la deuda podía tomar la forma de títulos y derivados financieros, y estos podían tomar la forma de nuevas deudas por la expectativa compartida, entre compradores y vendedores, de su convertibilidad en dinero.

La cadena de metamorfosis ocurrida "socializó" al capital de una forma inesperada: a través del intercambio vinculó al capital productivo con un número creciente de capitales financieros conectados entre sí, en una intrincada red de relaciones que hizo su destino más colectivo que nunca. Concentró y centralizó también los capitales financieros y productivos haciendo que el destino de todo el sistema dependiera de la suerte de unos cuantos nodos que condensaban una mayor cantidad de vínculos de activos y obligaciones del sistema en su conjunto.

Subyacente a ese destino común hubo un efecto muy poco visible de la cadena de metamorfosis desatada por la creatividad financiera del capital. Los nuevos derivados y títulos financieros hicieron parte de la ola de innovaciones financieras resultante de la desregulación iniciada en los años 1980. La metamorfosis de las deudas en derivados y títulos financieros, y de estos en deudas, en intrincadas secuencias que generaron redes interdependientes de activos y obligaciones, indujo la emergencia de distintos tiempos del capital, articulados en un sistema complejo, cuyos efectos dinámicos intenta dilucidar este artículo.

He aquí la intuición inicial. Consideren las deudas hipotecarias en las que incurrieron cientos de miles de hogares en Estados Unidos, España, Irlanda y otros países. Las deudas hipotecarias originales tienen un tiempo medible en años, puntuado por eventos que vienen de los mercados: cambios en la diferencia entre el precio de las viviendas y el valor de la deuda, pago o impago de las cuotas por parte 
de los deudores. Es un tiempo "lento", solo sometido a los sobresaltos que pueden venir de la incertidumbre inducida por la operación del sistema capitalista en su conjunto, o por cambios en los ingresos o en la capacidad de pago de los deudores ${ }^{1}$.

El tiempo de las deudas hipotecarias y de los mercados de vivienda no es simultáneo con el tiempo asociado a los eventos de los mercados financieros en los que se intercambian o valoran los activos derivados de las deudas originales. Los mercados financieros están puntuados por eventos como la emergencia y el estallido de burbujas, la retroalimentación positiva de los precios de los activos financieros y las ventas a precio de quema, entre otros. ¿Pertenecen esos dos tiempos a un tiempo homogéneo único, el tiempo de los relojes que todos aceptamos en principio como "el tiempo" sin más? Tampoco: ambos tiempos, aunque entrelazados, no pertenecen a un tiempo homogéneo único, por la sencilla razón de que ese tiempo único no es compatible con los eventos que conforman los tiempos del capital.

¿Cómo es posible que esos dos tiempos no pertenezcan a un mismo tiempo homogéneo si los eventos asociados a los dos están situados en la misma época? Bastaría, al parecer, con verificar las fechas de las grandes oleadas de hipotecas nuevas y las de la creación de los nuevos derivados financieros para comprobar que están situadas en un mismo periodo histórico: 2000-2008. Pero esta comprobación solo garantiza que ambos tipos de intercambios mercantiles y monetarios fueron realizados en el mismo periodo de tiempo calendario, o tiempo de los relojes, y no refleja el flujo específico de eventos asociado a cada uno de ellos.

Lo que conduce al problema de la simultaneidad de los eventos en el tiempo. Una de las propiedades del tiempo absoluto de la mecánica clásica es la simultaneidad de los eventos. En ese marco teórico, la simultaneidad es alcanzada suponiendo la existencia de un único reloj que marca el tiempo para todo el sistema. Einstein propuso un cambio en las condiciones de estudio del problema.

Usamos muchos relojes sincronizados en lugar de uno, para poder así determinar cuándo dos sucesos distantes son simultáneos en un sistema de coordenadas; lo que se comprobará si los relojes sincronizados indican la misma hora en el instante en que se producen los sucesos (Einstein en Hawking, 2008, p. 423, cursivas añadidas).

¿Qué ocurriría, se preguntó, “cuando dos grupos de relojes se mueven uniformemente, uno en relación con el otro?” (ibíd., p. 424). Lo

${ }^{1}$ De hecho, en periodos normales, las compañías aseguradoras cubren los riesgos derivados del no pago de una fracción marginal de los deudores. 
que encontró es bien conocido: los relojes pueden alterar su marcha cuando están en movimiento. La simultaneidad, asumida como un postulado por la mecánica clásica, desaparecía en el contexto del experimento mental propuesto por Einstein. La clave del hallazgo de Einstein está en el papel de los sucesos, o eventos, en la construcción de su concepto del tiempo.

E1 tiempo absoluto solo es posible si todos los eventos del "presente" son registrados de manera simultánea por todos los relojes del sistema. Y si todos los eventos ocurridos pueden ser ordenados de acuerdo a un "antes" y un "después" de conformidad con lo que marcan los relojes en forma simultánea.

Veamos ahora lo que ocurre con las deudas hipotecarias y los mercados financieros donde se compran y venden activos y se valoran los títulos y derivados en que se han transformado las deudas. Los eventos relacionados con las deudas hipotecarias no son simultáneos con los eventos asociados a los mercados y derivados financieros. En los mercados financieros, los eventos reflejan las expectativas cambiantes de los agentes y las interacciones entre miles de ellos en un tiempo continuo que puede ser interrumpido por el estallido de burbujas, las caídas bruscas de precios o las ventas a precio de quema.

En estos mercados ocurren dos tipos de eventos, con escalas distintas: los precios cambian segundo a segundo, siguiendo distribuciones de martingala, pero también pueden sufrir variaciones muy grandes, de probabilidad muy pequeña pero conocida, formando las colas "gruesas" de sus distribuciones de probabilidad. Los tiempos en los que ocurren los dos tipos de eventos no son simultáneos entre sí ni con respecto a los eventos asociados a las deudas hipotecarias ${ }^{2}$.

Son eventos distintos, que ocurren en "momentos" distintos, puntuados por ritmos distintos. Pero no dejan de estar entrelazados: el valor de los derivados financieros (p. ej., de las obligaciones de garantía de deuda, CDO por sus siglas en inglés) depende del flujo de pagos de las deudas hipotecarias que les sirvieron como base para su emisión. Es una relación que no es ni directa ni determinística: es compleja $\mathrm{y}$ depende de las interacciones ocurridas en el sistema complejo que

2 Para los compradores de derivados financieros, el estado de las deudas hipotecarias era opaco, invisible de hecho: no tenían ninguna información sobre su estado, salvo la calificación que les dan las agencias calificadoras de riesgo. Como planteó Gorton (2010) se trataba, por definición, de títulos y activos insensibles a la información. En el contexto de la crisis global de 2008, los mercados financieros no cumplían la tarea que Fama (1970) postuló que hacían a la perfección: condensar toda la información concerniente a los activos financieros transados en las bolsas de valores. 
conforman. El entrelazamiento de tiempos distintos es la consecuencia del entrelazamiento entre fases distintas del capital propiciado por la metamorfosis de deudas en títulos financieros.

Para entender el entrelazamiento entre el tiempo propio de las CDO y el tiempo de las deudas hipotecarias subyacentes es necesario introducir una definición intuitiva de las obligaciones de garantía de deuda. Una CDO es una apuesta que hacen los inversionistas contra el riesgo de no pago de una deuda respaldada por un activo. También puede definirse como una promesa de pagar a los inversionistas, en una secuencia acordada de antemano, el flujo de efectivo que el título recibe del conjunto de bonos y otros títulos asociados a la CDO. No es una apuesta directa. Su concreción requiere de varios pasos. Primero los activos (deudas) creados por la compañía originaria (el banco hipotecario, p. ej.) se venden a un intermediario (llamado vehículo de propósito especial) que emite unos títulos en el mercado de capitales con cuya venta se financia la compra de los activos (deudas) iniciales.

Segundo, las deudas compradas son "cortadas" en franjas o secciones de acuerdo a la certeza asociada a su pago: las más seguras son denominadas AAA, las que no son tan seguras $\mathrm{AA}$, las siguientes $\mathrm{A}$, $\mathrm{BB}, \mathrm{B}$, en orden descendente de seguridad, hasta llegar a las menos seguras de todas, las residuales. Si algunos préstamos dejan de pagar, los residuales serán los primeros en sufrir la pérdida correspondiente, mientras que los más antiguos, o AAA, serán los últimos en sufrir una pérdida. La tasa de interés está asociada en forma inversa a la seguridad o riesgo de cada franja.

Tercero, distintas franjas de deuda son agrupadas en una CDO para redistribuir el riesgo, incluyendo combinaciones de deudas seguras, no tan seguras y muy inseguras en un único conjunto. Ese conjunto inicial puede ser transformado en otro y en otros, denominados $\mathrm{CDO}^{2}$, o $\mathrm{CDO}$ de CDO. E1 supuesto subyacente es el de independencia de los eventos de no pago: el no pago de una deuda es independiente del no pago de las demás. La implicación fundamental sobre el tiempo está en la posposición y socialización del pago de la deuda realizada por su transformación en derivados que pueden ser transformados en nuevos derivados en procesos que solo son detenidos por la emergencia de orden en la forma de una crisis.

Las metamorfosis de deuda en derivados financieros y de estos en deudas van más allá de la simple circulación de mercancías: implican la generación de vínculos adicionales entre capitales financieros y productivos y su entrelazamiento en una red interdependiente más 
grande de procesos de circulación y producción del capital, en la forma de activos y obligaciones, que antes no estaban conectados en términos de su conversión potencial en dinero ${ }^{3}$.

En la primera década de este siglo, la conectividad creciente entre capitales financieros y productivos generó una red de activos y obligaciones con una dinámica propia derivada de las relaciones cambiantes entre los distintos nodos (capitales) que la conformaban. El tiempo asociado a la red global de obligaciones y activos financieros es distinto a los tiempos ya definidos. Es un tiempo relacional, constituido por los eventos que ocurren en la topología de la red. Son los eventos colectivos resultantes de las relaciones entre miles de agentes, en un mundo financiero global, incluida la posibilidad de cambios de estados o de fase, lo que constituye el tiempo relacional de las redes financieras interconectadas.

Esas redes tienen la propiedad de producir orden mediante el cambio de estado o de fase de la red, originada en su interconectividad y en la emergencia local de algún tipo de vulnerabilidad, casi siempre derivada de la activación de mecanismos de retroalimentación positiva. Si hay un número suficiente de nodos interconectados que hayan devenido vulnerables por sus relaciones con otros nodos vulnerables es probable que emerja orden en la forma de una crisis global. El tamaño del componente interconectado vulnerable determina la magnitud de la crisis global. Entre más grande sea y en la medida en que integre a los nodos más importantes y conectados de la red global, mayor será la magnitud de la ruptura ocasionada.

En la distribución de probabilidad de los eventos de la red financiera global, la probabilidad de un evento de gran magnitud es muy pequeña, pero conocida. Lo que no se conoce es cuándo ocurrirá. El carácter relacional del tiempo de la red tiene una contrapartida en el tamaño de los eventos que ocurren en ese tipo de red: son eventos de gran tamaño. Las denominadas cascadas globales (o crisis global en nuestro caso) son eventos muy poco probables, pero de gran magnitud (Watts, 2002). Su tamaño está relacionado con la magnitud de la conectividad existente y el grado de vulnerabilidad derivado del

${ }^{3}$ François Chesnais (2017) muestra que a pesar del papel crucial de los derivados financieros en el desencadenamiento de la crisis financiera global, las transacciones de productos derivados representan hoy 8 veces el PIB mundial. Confirma también la altísima concentración de los productos derivados: en 2016, el 90\% de su emisión en Estados Unidos estaba concentrado en 4 grandes bancos: JP Morgan, Bank of America, Citibank y Goldman Sachs. 
apalancamiento creciente generado por el proceso de metamorfosis de deuda en derivados financieros y de estos en deuda ${ }^{4}$.

Cuando esto ocurre, la red "condensa" los diversos tiempos generados por la metamorfosis de deuda en activos y de activos en deuda, generando una crisis de gran magnitud. El entrelazamiento de los distintos tiempos, casi siempre fuera de sincronía, deviene sincronizado por la convergencia precipitada por el proceso de percolación, resultante de la existencia de trayectorias conexas de nodos (capitales) que atraviesan todo el sistema. La vulnerabilidad derivada del no pago de los deudores de hipotecas "se propagó" a todo el sistema capitalista global a través de las trayectorias de conexiones creadas por el entrelazamiento de deudas y derivados financieros en redes interdependientes.

\section{METAMORFOSIS DE LA DEUDA Y DERIVADOS FINANCIEROS}

La oleada de innovaciones financieras aparecidas en las dos últimas décadas ha sido interpretada, o como una maniobra más del capitalismo financiero improductivo, o como un índice de la pobreza analítica de la teoría económica ortodoxa, o como una combinación de las dos, fundamentada en la codicia irrefrenable de Wall Street. Las tres ignoran una interpretación más simple: la oleada de innovaciones financieras no es más que una extensión, al proceso del crédito, de la metamorfosis de las mercancías en la forma monetaria del valor. En vez de que las mercancías tomen la forma de valor dinero, el crédito (la deuda) es el que toma, en potencia, la forma monetaria del valor. La sustitución de deuda por activos financieros que actúan como garantía de esas deudas y permiten incurrir en nuevas deudas, que reclaman nuevos activos como garantía de pago, genera una compleja cascada interdependiente de deuda, garantías de pagos y activos. Las consecuencias son decisivas para entender la dinámica reciente del capital financiero global.

¿Cuál es el papel de los derivados y demás activos financieros? Consideremos otra vez las $\mathrm{CDO}^{5}$. Al convertirse en CDO, la deuda

${ }^{4}$ Lo que no excluye la existencia de cascadas pequeñas. Pero las cascadas globales no son cascadas pequeñas que se vuelven grandes. Son un fenómeno distinto, consustancial a las redes globales en las que se cumplen ciertas condiciones; ver Watts (2002) y Gai y Kapadia (2012).

${ }^{5}$ La lista de deudas o créditos convertibles en títulos o activos financieros es larga y creciente. Incluye, por ejemplo: préstamos para la compra de vehículos, créditos estudiantiles, hipotecas, alquiler de aviones, alquiler de vehículos, flujos de caja de propiedad intelectual y muchos más (Gorton, 2010, p. 22). 
adquiere ahora la forma monetaria del valor. Pero lo hace en términos potenciales. La posposición de pago de la deuda toma la forma de cadenas de títulos o activos que pueden convertirse eventualmente en la forma monetaria del valor. Quienes invierten en esos títulos esperan obtener ganancias monetarias derivadas de la posesión de capital y del riesgo asumido en las apuestas realizadas. Es "capital fetiche", capital que se relaciona consigo mismo para producir ganancias, en un contexto de riesgo interdependiente y de posposición del pago de la deuda. El fetichismo del capital aparece aquí en todo su esplendor:

Lo que obtenemos, por tanto, es la forma fetiche del capital y la concepción de capital fetiche. En D-D' tenemos una forma sin sentido del capital, la perversión y la objetivación de las relaciones de producción en su grado más alto, la forma portadora de interés, la forma simple del capital que antecede su propio proceso de reproducción. (Marx, 1967, cap. 24, p. 392)

Con el desarrollo de las innovaciones financieras, la adopción de esta forma alcanzó consecuencias sistémicas. Las cadenas de pares de acreedores y deudores, y de emisores de títulos y compradores de ellos, conforman una red interconectada de capitales que se extiende por todo el mundo, y ejerce de hecho la mediación social entre los flujos de pago de la deuda original y el valor de las obligaciones. La competencia entre capitales adquiere la forma de una compleja cooperación social no planeada: la suerte de cada uno de los capitales depende de la suerte de los demás de acuerdo con sus interrelaciones en los mercados financieros ${ }^{6}$.

Si el crédito pospone el momento de la crisis, los derivados financieros posponen una y otra vez la posibilidad de la crisis en el tiempo, separando compradores y vendedores, o deudores y acreedores, y sustituyéndolos, en una secuencia que se prolonga en el tiempo, por otras secuencias de compradores y vendedores que no conocen nada con respecto al estado de la deuda original.

Esa secuencia es infinita en principio: si no fuera así el capital no sería capital. Sin embargo, y esta es la clave de la dinámica del capital, deja de serlo en el momento de la crisis. No es posible alargar para siempre la sustitución de los pares iniciales de compradores y vendedores por los múltiples pares interconectados que intercambian las

${ }^{6}$ Nótese que estos activos financieros dependen de los flujos de efectivo que los deudores originales están pagando. Nótese también la transformación de las deudas originales (que son pagadas o no pagadas en efectivo) en activos que son, a su vez, dinero potencial y están en la posición de la forma equivalente del valor. 
obligaciones y títulos of recidos en el mercado. La eventual emergencia de orden lo impide. En algún momento la posibilidad de no pago se hará real, y la información sobre su ocurrencia, así como la exigencia de la conversión inmediata de todas las deudas en dinero, llegará a los poseedores de las obligaciones respectivas a través de las miles de trayectorias existentes.

Al posponer la crisis en múltiples transacciones se multiplica en forma exponencial el tamaño de la crisis pospuesta. Cada vez que una obligación es intercambiada y que nuevas obligaciones son emitidas y aprobadas por las firmas calificadoras de riesgo, el tamaño de la crisis potencial crece: lo que era una deuda sencilla entre un banco hipotecario y un comprador de vivienda se convierte en una cascada de obligaciones que involucran múltiples compradores y vendedores interconectados en todo el mundo.

El mecanismo subyacente involucra posponer la auto-corrección y ajuste del precio de las obligaciones al estado de las deudas originales. Pero esa auto-corrección no es automática y no evita la crisis. No es un mecanismo de retroalimentación negativa que conduce al equilibrio de forma automática. Por el contrario, al igual que ocurría con el crédito simple, es un mecanismo de retroalimentación positiva que conduce a la crisis: la destrucción masiva de valor hasta alcanzar un equilibrio temporal y recomenzar el proceso de acumulación.

\section{CRÉDITO Y DINERO}

El papel del crédito es posponer la ocurrencia de la crisis. Este papel se puede ver en los diagramas de circulación del capital de Marx. Mientras que el fin de la circulación de mercancías (M-D-M) es la adquisición de una mercancía como valor de uso, la circulación del capital requiere que el dinero devenga dinero acrecentado (D-M-D'). Las diferencias en su disposición no son secundarias: la primera comienza con una venta y termina con una compra; la segunda comienza con una compra y termina con una venta (Karatani, 2005).

En ambas está abierta la posibilidad de que la segunda parte de la cadena no ocurra: la mercancía podría no ser comprada en la primera, y podría no ser vendida en la segunda. Las implicaciones son distintas. Si el circuito mercantil se interrumpe, algunas (o muchas) mercancías quedarán sin valor. Si la segunda parte del circuito del capital no tiene lugar, no habrá reproducción ampliada de ese capital, y el tamaño de la crisis y su ocurrencia dependerán de que eso mismo le ocurra a un número mayor de capitales. 
E1 capital dispone del crédito como mecanismo permanente de posposición del momento de la venta y, por tanto, de la crisis. El crédito pospone el acto de la venta M-D', entregando dinero a cambio de la mercancía (D-M), antes de que la venta final haya ocurrido ${ }^{7}$. La posposición de la venta final M-D' lleva consigo la posibilidad de que ese evento no ocurra en el futuro. En el momento del crédito, las dos partes coinciden en creer que la mercancía subyacente se va a vender en el mercado. Pero también coinciden en que es probable que no se venda, o que se venda a un valor menor del esperado. Es lo que Karatani, siguiendo a Marx, denomina la posibilidad de la crisis. Esa posibilidad es un evento probabilístico con unas características especiales: sabemos que va a ocurrir, podemos conocer incluso su magnitud, pero no sabemos cuándo va a ocurrir.

La incertidumbre abierta por la posibilidad de no venta de la mercancía, o de no pago de la deuda, es pospuesta, introduciendo al tiempo en el escenario. Lo que era un problema concerniente a las dos partes individuales de la transacción original se convierte en un problema del sistema de intercambio monetario y crediticio, en la medida en que más individuos recurren al crédito y posponen el momento potencial de la crisis. El crédito no hace más que posponer la incertidumbre y acumularla de acuerdo con la posibilidad de la coincidencia temporal de muchos eventos de no pago ${ }^{8}$.

¿Hasta cuándo puede ser pospuesto el momento de la venta o del pago del crédito? Hasta el momento en que los activos producidos por el sistema financiero para sustituirlos y posponer el momento del pago de la deuda tengan que ser pagados en dinero. En el ínterin, el crédito, el apalancamiento de la deuda y las expectativas optimistas generalizadas generan burbujas en distintos sectores de la economía. Las burbujas separadas pueden llegar a agruparse cuando el sistema se acerca a su punto crítico y ocurre un cambio de estado.

Siguiendo el análisis de Karatani (2005) y de Marx es posible detectar un crecimiento vertiginoso del número de vendedores que evitan estar en la difícil posición de vender. Los bancos hipotecarios originales vendían los préstamos a bancos de inversión que a su vez

7 Al hacerlo está creando dinero: el crédito es una adición a la cantidad de dinero en la economía.

${ }^{8}$ Pero esa coincidencia no es producto ni de la suerte ni del azar. Es el resultado de la operación del sistema interdependiente de activos financieros, obligaciones y garantías de pago, y sus transformaciones. Su dinámica puede ser aproximada mediante el uso de las redes complejas y la teoría de la percolación (Solé, 2011). 
los vendían a múltiples inversionistas en la forma de derivados financieros (Gorton, 2010).

Mientras que las expectativas con respecto al precio de las obligaciones sean optimistas, el número de compradores crecerá y lo mismo hará el precio de los activos. En el ínterin, el valor asociado a las obligaciones crecerá muy rápido. Cuando los compradores deban volver a la posición de vendedores el tamaño del valor involucrado en las transacciones por "cerrar" será mucho más grande. Cuando comiencen a vender, el deseo de desprenderse de unos activos que están perdiendo valor hará que su valor caiga mucho más rápido y la destrucción de valor alcanzará proporciones gigantescas.

De hecho, las crisis del capitalismo solo pueden ocurrir con el desarrollo del sistema de crédito y bancario. No es posible una crisis puramente "real" del capitalismo:

Las crisis reales solo pueden ocurrir después del desarrollo de los sistemas de crédito en la economía mercantil. El crédito consiste en cerrar la venta de una mercancía, pero posponiendo el arreglo de cuentas [...] Una crisis comienza cuando algo desata la comprensión de que la compra y la venta suscritas por el crédito en realidad no tienen lugar. En ese sentido, todas las crisis toman la forma de crisis de crédito. (Karatani, 2014, p. 202)

Marx vio el papel decisivo de "la superestructura del crédito" en la transformación del capitalismo y en el paso de un sistema basado en capitales individuales a un sistema interdependiente de crédito, en el que el riesgo deja de ser individual para convertirse en colectivo:

[E1] crédito ofrece al capitalista individual, o a quien es considerado un capitalista, control absoluto dentro de ciertos límites sobre el capital y la propiedad de otros, y por tanto sobre el trabajo de otros. E1 control sobre el capital social, no sobre su capital individual, le da control sobre el trabajo social. El capital mismo, lo que un hombre realmente posee o se supone que posee a los ojos del público, deviene puramente una base para la superestructura del crédito. [...] Lo que el mayorista especulador arriesga es propiedad social, no su propiedad. (Marx, 1967, pp. 438-439, cursivas del original)

En el capitalismo financiero global de hoy, el mecanismo básico de la crisis es el mismo, pero no lo son ni su tamaño ni su estructura ni sus trayectorias. Las crisis ocurren hoy en estructuras interconectadas de capitales financieros y reales, susceptibles de bruscos cambios de estado. La posposición de la crisis generada por los derivados multiplica en forma exponencial la magnitud del valor involucrado en el no pago potencial de las deudas originales. Por eso, las claves de la evolución reciente del capitalismo no deben ser buscadas en las fluctuaciones de 
la producción real de mercancías, sino en la forma en que "la historia es organizada por la economía monetaria” (Karatani, 2005, p. 160).

El tamaño de la crisis deviene mucho mayor que lo esperado por el más pesimista de los críticos. De hecho, el tamaño de los créditos hipotecarios en la crisis global de 2007-2009 -la parte "real" de la crisis- fue solo una proporción mínima de la destrucción total de valor ocurrida. La explicación de esa multiplicación gigantesca del valor destruido está en el crecimiento del número y la magnitud de los capitales interconectados a través de la red de activos y obligaciones generada por los procesos de metamorfosis descritos arriba.

Sin embargo, no se trata de un simple problema de circulación de mercancías. El problema ahora es la valorización del capital en su conjunto. Los múltiples vínculos entre capitales financieros y productivos, generados por los procesos de metamorfosis sugeridos, hacen que la valorización del capital no dependa ahora en forma exclusiva de su tasa de ganancia, sino del estado de sus interrelaciones con los demás capitales, en términos de pago o no pago de las obligaciones adquiridas, y de la valorización esperada de los capitales atados a ese tipo de inversiones.

E1 circuito original M-D-M se convierte ahora en D-M-D': el dinero se convierte en mercancía para volver a su condición de dinero, con la expectativa de que la cantidad que regresa sea mayor que la original. La transformación del circuito cambia también las condiciones de la posibilidad de crisis. Ya no se trata de crisis de sobreproducción o de falta de ventas de mercancías o de debilidad en el consumo. El problema ahora es que el capital no regrese valorizado a su punto original, que no crezca en valor. Es decir, que no realice las expectativas de valorización de los inversionistas. No es una crisis de sub-consumo o sobreproducción sino de insuficiente valorización del capital. O, como veremos más adelante, de exceso de liquidez o de capital en potencia.

La separación del proceso de valorización del capital en dos partes distintas y asimétricas hace que los participantes no sepan lo que ocurre en la otra parte. Pero esas dos partes, invisibles la una para la otra, vuelven a unirse en ciertos momentos críticos. El lenguaje de Marx en este punto es similar al de los procesos de percolación en la física contemporánea del estado sólido:

La circulación estalla a través de todas las barreras espaciales y temporales impuestas por el intercambio directo de los productos, y lo hace separando la identidad directa presente, en este caso, entre el intercambio del producto de uno mismo y la adquisición del producto de otro 
en dos segmentos antitéticos de venta y compra. Afirmar que estos procesos, mutuamente independientes y antitéticos, forman una unidad interna es afirmar también que su unidad interna se mueve hacia delante a través de antítesis externas. Esos dos procesos carecen de independencia interna porque se complementan el uno al otro. Por lo tanto, si la afirmación de su independencia externa procede hasta cierto punto crítico, su unidad se hace sentir violentamente al producir una crisis. (Marx, 1976, p. 209, cursivas añadidas)

Si la separación entre la compra y la venta es una condición de la circulación del capital, su unidad solo puede ocurrir en un punto crítico: aquel donde se encuentran en forma violenta múltiples enlaces de compra y venta, y cambia el estado del sistema capitalista. Tanto la organización del sistema, en una estructura conexa en la que todos sus elementos están conectados entre sí, como la existencia de un punto crítico donde el sistema cambia de estado en forma brusca y repentina son propiedades compartidas por las dinámicas del capitalismo financiero global y de los sistemas físicos complejos.

Cuando un sistema, físico o económico, es atravesado por una cadena interconectada de nodos vecinos que pueden coordinarse en una misma acción o arribar a un estado similar, estamos ante un proceso de percolación. Esos procesos ocurren en sistemas organizados en forma de red: estructuras compuestas por nodos unidos por vínculos que representan algún tipo relación; en el caso del capitalismo financiero, activos y obligaciones. La crisis financiera global de 2007-2009 tuvo como plataforma el sistema financiero global de obligaciones y activos interconectados.

Se trata de una crisis de valorización del capital: la corrección violenta que el sistema impone de la posposición, en apariencia infinita, del crédito y la deuda, en un contexto de alta interconexión de los capitales. Es también el momento en que las expectativas de deudores y acreedores cambian en forma brusca y generalizada. Este momento se registra en el tiempo de los relojes como "la fecha del calendario" en que ocurrió una crisis financiera global.

Las narrativas de la crisis reciente fijan la caída de Lehman Brothers -15 de septiembre de 2008- como la fecha en que se desató la crisis financiera global'. Cuando se habla en términos "más

9 James B. Stewart (2009) contó, para The New Yorker, los eventos que condujeron a la quiebra de Lehman Brothers y a la crisis financiera global. Entre el viernes 12 y el viernes 19 de septiembre de 2008, los bancos de inversión y seguros más grandes de Estados Unidos y del mundo, de un lado, y el gobierno de Estados Unidos, del otro, negociaron la salvación del capitalismo global y de ellos mismos. Si bien los ocho días narrados por Stewart reflejan lo ocurrido en Manhattan, no son iguales a lo ocurrido en el tiempo 
históricos" se alude a la crisis de 2007-2009, o a la crisis iniciada en 2007 y a la recesión sin terminar que la siguió. Ninguna de esas narrativas tiene en cuenta la sincronización violenta de los distintos tiempos del capital propiciada por la topología y la conectividad de la red subyacente.

\section{EMERGENCIA DE LA RED: CONECTIVIDAD, CRÉDITO Y CAPITAL FINANCIERO}

En el capitalismo financiero, la posposición del momento de la venta toma la forma de múltiples y diversos activos, obligaciones y derivados, vía las metamorfosis basadas en la forma monetaria del valor. La deuda original es transformada en distintos activos, de formas y diseños diversos, sobre la base de su convertibilidad esperada en dinero. Lo nuevo y crucial es que esa transformación toma ahora la forma de una red creciente de obligaciones y activos que se extiende por la economía global y compromete al capital en su conjunto. Dado que el capital ha tomado la forma de una red compleja, su estructura y dinámica se deben tratar entonces de la misma manera que se tratan las redes complejas.

Cuando un capitalista financiero emite un título o un activo y este es comprado por otro banco o firma, aparece un vínculo entre los dos, en el que el primero se compromete a convertir en dinero la obligación que tiene con el comprador de su título, y el segundo paga una cierta cantidad de dinero a cambio del activo emitido por el primero. Este tipo de vínculo se establece todos los días, en distintas magnitudes, a lo largo y ancho de la economía financiera global. Las posiciones (activos menos obligaciones) de cada uno de los capitales financieros dependen de la "salud" de las posiciones de los demás capitales y de las interconexiones entre todos los capitales de la red compleja que los une. Su suerte no es independiente: depende, en principio, del estado de sus vecinos, y en situaciones de crisis del estado de la red global de interconexiones financieras.

Con la conversión de deudas en activos financieros y su garantía con nuevos títulos que a su vez generan nuevas deudas, apoyadas en nuevos títulos, el tamaño y la complejidad de la red financiera resultante ha crecido en forma vertiginosa, desencadenando dinámicas que involucran burbujas, y cambios de estado masivos y bruscos. La

del capital. Los grandes banqueros y los líderes económicos del gobierno de Estados Unidos trataban de salir de la manera menos costosa posible de una crisis que ya era inevitable. El tiempo del capital difiere del tiempo narrativo de las negociaciones alrededor de un evento que ya estaba ocurriendo. 
crisis inherente al capitalismo es ahora una propiedad emergente de la dinámica asociada a la compleja red financiera global creada en las últimas décadas.

\section{Gráfica 1}

La red financiera interdependiente

Generada por la conversión de deuda en derivados y de estos en deuda

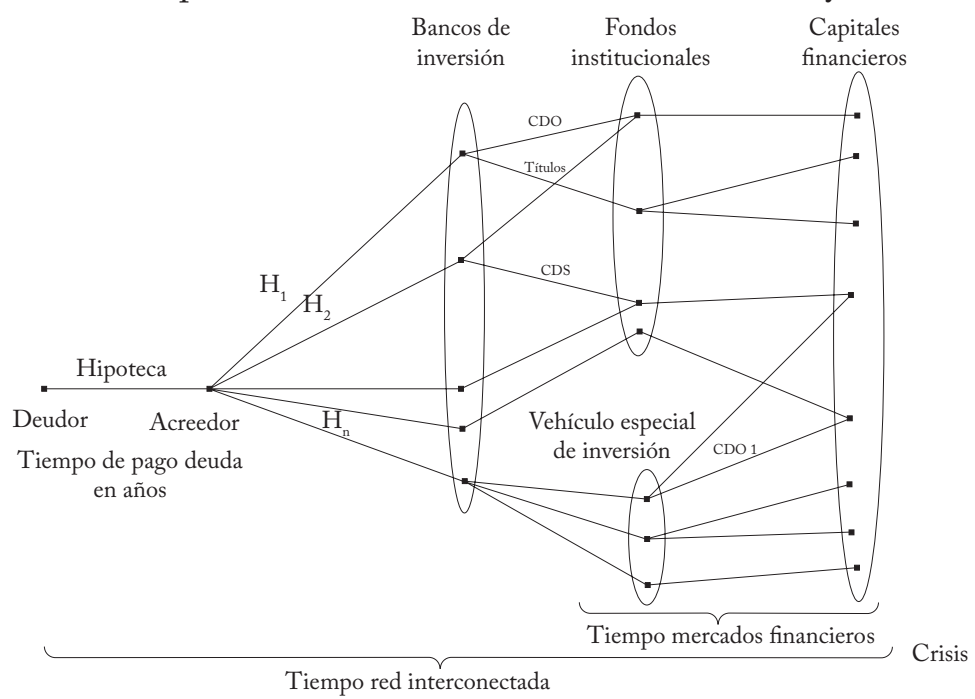

$\mathrm{H}_{1}, \mathrm{H}_{2}, \ldots, \mathrm{H}_{\mathrm{n}}$ : Hipotecas; $\mathrm{T}$ : títulos. Los puntos son nodos (capitales financieros), las líneas son los vínculos (obligaciones y activos compartidos por pares de nodos). La red no representa el tamaño real de los distintos tipos de capital financiero. La asimetría de los tamaños tampoco representa la asimetría existente en el mundo real.

En la red interdependiente de activos y obligaciones ocurren procesos de contagio entre capitales vecinos que propagan la falta de pago o la vulnerabilidad de la posición financiera de un subconjunto de nodos a las posiciones financieras de todos los nodos conectados a ellos mediante trayectorias formadas por los vínculos presentados más atrás. Si el contagio o la vulnerabilidad general alcanzan cierto punto crítico, podría ocurrir una crisis sistémica o cascada global (Gai y Kapadia, 2010; Watts, 2002). De igual modo, el crecimiento excesivo del precio de ciertos activos financieros, sostenido por expectativas optimistas, puede conducir, vía retroalimentación positiva sobre la demanda, a burbujas de precios que, eventualmente, estallarán.

Los efectos del no pago de obligaciones y la caída del precio de los activos en algunas vecindades de la red pueden extenderse a otras vecindades a través de trayectorias conexas resultantes de los vínculos conformados por activos y obligaciones. Si en algún momento emerge 
una trayectoria conexa de la dimensión de la red, la vulnerabilidad presente en algunas vecindades se extenderá a un componente grande de la red generando una "cascada" o crisis financiera global. Es el momento en el que todos los capitales quieren convertirse en dinero y sus agentes quieren vender, a cualquier precio, sus activos, derivados y obligaciones. En el lenguaje de los sistemas complejos se trata de un cambio de estado o de fase del sistema, que ocurre cuando la conectividad del sistema alcanza un cierto umbral.

La aplicación de las redes complejas al estudio de la dinámica de las redes financieras globales ha permitido establecer que:

Choques macroeconómicos y los efectos de la retroalimentación de los precios de los activos se entremezclan para generar colas gruesas en estas distribuciones [de probabilidad] y mostrar que rupturas financieras de gran escala son posibles. (Anand et al., 2012, p. 27)

Las crisis financieras globales conducen a la destrucción masiva de valor y de capitales y a una concentración y centralización del capital aún más extrema. Marx ya había detectado las relaciones profundas y poco visibles entre el crédito y la centralización del capital:

En sus primeras etapas, el sistema [de crédito] aparece furtivamente como un humilde asistente de la acumulación, llegando a las manos de capitalistas individuales o asociados mediante hilos invisibles de recursos monetarios, que yacen esparcidos en mayores o menores cantidades sobre la superficie de la sociedad; pero pronto deviene un arma nueva y terrible en la batalla de la competencia y es finalmente transformada en un enorme mecanismo social para la centralización de los capitales. (Marx 1976, p. 778, cursivas añadidas)

\section{LA DEUDA Y EL TIEMPO DEL CAPITAL}

En la red financiera global ocurren múltiples eventos. Burbujas, estallidos de burbujas, contagios, crisis globales, emisión de activos y títulos, innovaciones financieras, quiebras, ventas a precio de quema, y todos ocurren como consecuencia de las dinámicas propias de las redes financieras interdependientes. Una tentación inmediata es postular que el tiempo es la fuente de su ocurrencia. La teoría de la relatividad general de Einstein ha propiciado el cuestionamiento de esa concepción "natural” y de sentido común del tiempo.

En un artículo en homenaje a Einstein, Kurt Gödel sugirió una implicación, ignorada hasta ese momento, de la teoría de la relatividad general: la simultaneidad del tiempo es relativa. También lo es la sucesión de eventos constitutivos de la concepción del tiempo aceptada por todos. Para llegar a su sorprendente conclusión, Gödel 
partió de establecer una formulación lógica para el tiempo como sucesión y simultaneidad:

Se requiere al menos que cualquier par de eventos puntuales sean simultáneos o uno suceda al otro, por ejemplo, que la sucesión temporal defina un orden lineal completo de todos los puntos de eventos. Existe un orden parcial absoluto. (ibíd.)

Pero esa definición natural y lógica del tiempo enfrentaba una objeción lógica y física muy fuerte, derivada de la teoría de la relatividad general de Einstein ${ }^{10}$ :

La afirmación de que A y B son simultáneos (y para una clase grande de pares de eventos, también la afirmación de que A ocurrió antes de B) pierde su significado objetivo, en la medida en que otro observador, con el mismo grado de corrección, puede afirmar que A y B no son simultáneos (o que B ocurrió antes que A). (Gödel, 1949a, p. 557)

El tiempo como flujo ordenado de eventos devendría ilusorio, y la existencia misma de un lapso de tiempo objetivo dependería de la forma en que la materia en movimiento esté distribuida en el mundo. E1 hallazgo lógico y físico de Gödel da un punto de partida analítico para entender el problema del tiempo del capital. Intentaré hacerlo, una vez más, en el contexto propiciado por la deuda en un mundo financiero interdependiente.

Al analizar el tiempo del capital desde la perspectiva más integral del espacio-tiempo, David Harvey confirma la profunda divergencia entre las lecturas de Marx realizadas desde una perspectiva compleja y desde una perspectiva determinística y lineal:

Suponemos un marco temporal y espacial fijo y universal y lo usamos para localizar, ordenar y calibrar la actividad en ese marco. Esto es lo que proporcionan el tiempo de los relojes y los espacios medidos de Descartes y Newton apoyados por 1 geometría euclidiana [...] O aceptamos que el tiempo y el espacio se pueden conceptualizar y experimentar de múltiples maneras [...] Un roble internaliza cierta medida del espacio-tiempo cuando crece. Su medida es muy distinta de la que define el crecimiento del maíz [...] Incluso nuestra comprensión científica contemporánea parece inestable. Las nociones de espacio y tiempo en física han evolucionado: de las newtonianas, a través de la relatividad de Einstein, al espacio-tiempo relacional implicado en la mecánica cuántica de Niels Bohr. (Harvey, 2017, pp. 137-138)

${ }^{10}$ Gödel presentó ese mismo año la fundamentación física de su hallazgo lógico en otro artículo (Gödel, 1949b). Su argumentación básica planteaba que en mundos con materia en rotación y curvas cerradas de tiempo, en una de esas curvas el evento $\mathrm{A}$ precede a $\mathrm{B}$, mientras que en otra $\mathrm{B}$ precede a A, reviviendo la idea de un viaje en el tiempo, y cuestionando de nuevo el carácter simultáneo y sucesivo del tiempo. 
¿Es posible ordenar en forma secuencial los eventos asociados a la red financiera global y postular que ese orden corresponde al tiempo del capital ${ }^{11}$. ¿Pertenecen todos los eventos asociados a la red financiera a un mismo flujo de capas de "ahora", que en sucesión infinita constituirían el tiempo en el sentido que Gödel, siguiendo a Kant y Parménides, le da en su artículo? Si el tiempo es ilusorio, ¿de qué estamos hablando cuando hablamos del tiempo del capital? Lo que sigue es una respuesta tentativa a partir de una lectura del tiempo del capital de Marx desde una perspectiva compleja, y de un bosquejo de lo que podría ser el tiempo del capital en el contexto de la interconectividad global de las redes financieras.

El tiempo del capital en Marx no es lineal ni absoluto. No se puede ordenar como una secuencia de capas de "ahora" que constituirían en cada momento el presente del capital y, en su flujo secuencial, el tiempo absoluto del capital. En realidad, el tiempo del capital es una propiedad inherente a su estructura compleja. Louis Althusser fue uno de los primeros en detectar cuán distintas son las concepciones del tiempo de Hegel y Marx, y sus consecuencias filosóficas y teóricas.

No es posible, afirmó, entender la concepción del tiempo del capital de Marx aceptando, como muchos marxistas lo hacen aún hoy, la "categoría hegeliana de contemporaneidad del presente" (Althusser et al., 2015, p. 246, cursivas del original). ¿Por qué no? Porque el todo complejo del capital, en sus distintos niveles y estructuras

Ya no se puede pensar en la co-existencia del presente hegeliano, el presente ideológico en el que la presencia temporal coincide con la presencia de la esencia, y su fenómeno. En consecuencia, el modelo de un tiempo continuo y homogéneo que toma el lugar de la existencia inmediata, que es el lugar de la existencia inmediata de esta presencia continua, ya no se puede ver como el tiempo de la historia. (ibíd., cursivas del original)

Si la totalidad del capital no existe en el presente hegeliano, en el que la existencia inmediata es la realización de la esencia del fenómeno, ¿Cómo entender entonces el tiempo del capitalismo como totalidad? ¿Cómo leer su despliegue histórico? ¿Cómo localizar sus aconteci-

${ }^{11}$ El economista heterodoxo Ha-Joon-Chang sugiere una fórmula interesante: "Podríamos decir que los derivados son apuestas sobre cómo evolucionarán otras cosas con el paso del tiempo" (2015, p. 268, cursivas añadidas). En la definición de Ha-Joon hay dos perspectivas distintas. Una es subjetiva: los compradores de derivados apuestan acerca de cómo van evolucionar otras cosas (el pago de las hipotecas, p. ej.). La otra, al parecer objetiva: supone la existencia objetiva del paso del tiempo. Ambas, sin embargo, sugieren la existencia de tiempos distintos: hay cosas que evolucionan de cierta manera mientras el tiempo pasa. 
mientos y rupturas? La sugerencia de Althusser va en el sentido de reconocer la complejidad del tiempo del capitalismo en su movimiento, derivada de las interacciones complejas entre los distintos tiempos que lo conforman ${ }^{12}$. La lectura de El Capital

Muestra, por ejemplo, que el tiempo de la producción económica es un tiempo específico (que difiere de acuerdo al modo de producción), pero también que como tiempo específico, es un tiempo no lineal y complejo: un tiempo de tiempos, un tiempo complejo que no se puede leer en la continuidad del tiempo de la vida de los relojes, sino que se debe construir a partir de las estructuras peculiares de la producción [...] E1 concepto de este tiempo se debe construir a partir de la realidad de los diferentes ritmos que puntúan las distintas operaciones de producción, circulación y distribución. Por ejemplo, la diferencia entre tiempo de producción y tiempo de trabajo, la diferencia entre los distintos ciclos de producción (la rotación del capital fijo, el capital circulante, el capital variable, la rotación monetaria, la rotación del capital comercial y del capital financiero, etc.). (ibíd., p. 248, cursivas del original)

Las consecuencias de esta lectura del tiempo del capital como totalidad compleja son decisivas para entender el surgimiento de las crisis y la evolución del capitalismo global. Primero, no es posible leer el despliegue del capitalismo como el de una "sección esencial", un corte vertical común a todos sus niveles. Es decir, como lo que ocurriría en un presente homogéneo, en el que todos los eventos de todos los distintos tiempos del capital serían contemporáneos y pertenecientes a un mismo corte que los haría parte de un tiempo homogéneo.

Segundo, esa caracterización negativa no es suficiente. Lo que ocurre en realidad es algo más complejo y de más difícil tratamiento. Los tiempos del capital están entrelazados o interconectados, de modo que el problema ahora es entender los efectos de ese entrelazamiento. Althusser sugirió un camino para resolver ese problema a partir del tratamiento que Marx intuyó en El Capital:

$\mathrm{Y}$ este proceso, también, es lo que Marx denominó el entrelazamiento de los distintos tiempos (y aquí él solo menciona el nivel económico), el tipo de 'dislocación' y torsión de las distintas temporalidades, producida por distintos niveles de la estructura, cuya combinación compleja es lo que constituye el tiempo peculiar del desarrollo de su proceso. (ibíd., p. 252, cursivas del original)

${ }^{12}$ Walter Benjamin, siguiendo la interpretación de Hugo Fischer, sugirió una lectura similar para el tiempo de la tecnología en El Capital. En esencia, el tiempo de la tecnología no sería el tiempo de "la economía moderna, que [...] mide el tiempo de trabajo en términos del reloj" (Benjamin, 1999, p. 654). 
¿Cuáles son las consecuencias de esta concepción del tiempo del capital para el surgimiento de las crisis globales? Aquí aparecen dos lecturas alternativas de la posición de Marx con respecto al tiempo del capital y el surgimiento de crisis. Una lectura racionalista que postula la discordancia entre los distintos tiempos del capital como origen de sus crisis y una lectura compleja que interpreta las crisis del capitalismo como el orden emergente asociado a la sincronización de los tiempos del capital hecha posible por la expansión de las redes financieras interdependientes.

Aunque luzca paradójico, Daniel Bensaïd hizo ambos tipos de lectura. En su interpretación de la filosofía del encuentro de Althusser, después de subrayar la originalidad de la posición filosófica de Althusser con respecto a la concepción del tiempo en El Capital, concluyó que se puede leer ese libro como "un entrelazamiento de diferentes tiempos y descifrar sus crisis como un efecto de su discordancia" (Bensaï, 2007, p. 10) ${ }^{13}$.

Sin embargo, en un libro escrito y publicado poco después propuso una lectura compleja del tiempo en El Capital de Marx:

La continuidad de todo el conjunto del proceso depende, pues, de la discontinuidad y de la desincronización de los respectivos ciclos del capital monetario, del capital industrial, del capital comercial; es decir, del hecho de que el banquero puede extender su crédito al industrial, invertir antes de que las mercancías hayan sido agotadas por el comerciante, y de que el comerciante pueda tomar préstamos, para renovar su stock, antes incluso de que se le haya terminado el stock precedente. (Bensaï, 2012 , p. 125 , cursivas añadidas)

En la primera lectura el supuesto subyacente parece obvio: las crisis serían el efecto de la discordancia entre los distintos tiempos del capital. ¿Pero qué quiere decir que los tiempos del capital son discordantes? ¿Y cuál podría ser su relación con el "descifrar sus crisis" que sugiere Bensaïd? La discordancia parecería implicar que la falta de sincronía entre los distintos tiempos del capital es lo que llevaría a la ruptura violenta de los intercambios y la producción en el capitalismo. Mas, como vimos antes, es la posposición de la venta a través de la deuda, en distintos tiempos, lo que permite la continuidad del capitalismo.

$\mathrm{Su}$ segunda lectura, en cambio, intuía el carácter complejo del entrelazamiento de los tiempos de los distintos tipos de capital en una economía financiera. Pensando desde la lógica del volumen 2 de El Capital, Bensaïd vio que la reproducción del capitalismo requería

${ }^{13}$ Es posible que esta interpretación no sea más que un error de digitación. Los dos textos de Bensaïd a este respecto fueron escritos en el mismo periodo, y el más largo y preciso es el que introduce la segunda interpretación. 
la desincronización de los ciclos de los distintos capitales. Esa falta de sincronización estructural a las necesidades de reproducción y valorización del capital es lo que permitiría posponer la crisis. Por el contrario, y aunque Bensaïd no lo planteó en su texto, la sincronización brusca y violenta de los tiempos del capital es lo que conduce a la crisis global. Que no es más que la unidad entre procesos independientes inducida de forma violenta por la convergencia a un punto crítico, tal como lo planteó el propio Marx en El Capital.

A partir de esa premisa defenderé la tesis fundamental de este artículo: las crisis no son el efecto de la discordancia entre los distintos tiempos del capital. Son, por el contrario, el efecto de la sincronización entre los distintos tiempos del capital. Mientras se conserve la discordancia de sus distintos tiempos, el capital en su conjunto puede seguir operando gracias a la posposición del pago de la deuda y a la transformación de las deudas en múltiples activos financieros que interconectan los capitales a escala global.

La deuda pospone la venta y, por tanto, la ocurrencia de la crisis. Es una transacción que sustituye a un flujo monetario que debe ocurrir en algún momento en el futuro. Por tanto, el crédito y la deuda sitúan el intercambio y la valorización del capital en el tiempo. Es la posposición de la venta y la larga cadena de transacciones resultantes (apoyadas en nuevos activos y derivados financieros) la que constituiría, en principio, el tiempo complejo del capital en Marx.

Es claro que la forma monetaria del valor permite hacer apuestas sobre cómo evolucionarán las deudas con el paso del tiempo. Una deuda solo puede ser transformada en un derivado o en una CDO porque la relación asociada a la forma valor monetaria lo permite. La misma relación entre la forma relativa y la forma equivalente del valor reaparece en la transformación de la deuda en otros activos, derivados y productos financieros. Todos aspiran a convertirse en algún momento en dinero. $\mathrm{Y}$ todos guardan, en el momento de su transacción, una cierta relación de convertibilidad con la forma dinero. Pero su transformación final en dinero o liquidez solo ocurre en el momento de su maduración, o en el momento decisivo de la crisis: el momento en el que todos quieren tener dinero y nadie quiere mercancías.

Entre el momento de la primera transformación y el momento de la verdad, o de la conversión en liquidez, aparece el tiempo propio del capital. Es la secuencia de relaciones y transformaciones de las deudas iniciales la que produce el tiempo del capital financiero. No hay, entonces, un tiempo absoluto en El Capital de Marx: el tiempo es el 
resultado de la secuencia de relaciones y transformaciones propias de la operación interdependiente del capital.

Como no hay tiempo absoluto, hay distintas temporalidades correspondientes a distintas secuencias de eventos, a distintos tipos de relaciones entre capitales y a distintas dinámicas ocurridas en las redes financieras interdependientes. Por eso, a cada "nivel": "Le corresponde un tiempo propio [...] medido de manera específica [y] la especificidad de esos tiempos y de esas historias es diferencial" (Althusser et al., 2015, p. 247, cursivas del original).

El tiempo del pago de las deudas hipotecarias originales (medido en años) es distinto del tiempo de los mercados financieros, con sus procesos de retroalimentación positiva, el estallido de las burbujas correspondientes y sus cambios instantáneos en los precios de los activos. Y ambos son distintos del tiempo relacional de la red financiera global. Es evidente que los tiempos de maduración de los distintos tipos de deuda y de capital no están sincronizados en la mayor parte de los eventos que ocurren en la red financiera global. Es más: la falta de sincronización es lo que garantiza la posposición, en apariencia infinita, del pago de la deuda, y la operación continua del sistema capitalista en su conjunto ${ }^{14}$.

Una visión racionalista del capitalismo supone que la discordancia o falta de sincronización entre los distintos tiempos del capital es lo que lleva a las crisis, al desorden y a la destrucción masiva de riqueza. Por el contrario, es la emergencia de orden, derivada de la sincronización súbita de las decisiones de los agentes interconectados por redes financieras globales, lo que lleva a la crisis.

Axel Leijonhufvud condensa muy bien cómo los distintos procesos desfasados del capitalismo financiero terminan sincronizándose en el momento de la crisis:

La lenta acumulación del apalancamiento en la economía incrementa la conectividad de la red de deudas y obligaciones que se combina con el desfase de la madurez subyacente para hacer al sistema más frágil. Cuando el sector financiero eventualmente se mueva hacia el desapalancamiento, la conectividad del sistema pondrá distintos mecanismos de desviación y contrabalance en "sincronía". (Leijonhufvud, 2011, p. 6, cursivas añadidas)

Las metamorfosis derivadas de la forma monetaria del valor y de la posposición del crédito inducen la emergencia de tiempos distintos y entrelazados del capital y, a través de la formación de redes interdepen-

${ }^{14}$ Este artículo evita la discusión reciente sobre el tiempo en El Capital de Marx, en especial la interpretación del tiempo en sus distintos volúmenes. Para una revisión de esa literatura desde un punto de vista filosófico, ver Osborne (2008). 
dientes globales, la ocurrencia de crisis financieras de alcance global.Y es el carácter relacional del tiempo propio de la red interdependiente global lo que permite explicar la súbita sincronía que es la crisis.

\section{CONCLUSIÓN}

La crisis es el resultado de la convergencia de las distintas temporalidades (asociadas a distintos tipos de deuda y de su maduración) hacia un evento, y de la sincronización, vía conectividad, de distintos mecanismos de corrección de las desviaciones generadas por las burbujas financieras. Esos mecanismos destruyen riqueza y valor. $\mathrm{El}$ momento de la crisis no es uno más dentro de la sucesión de tiempo absoluto: es el punto crítico encontrado por la dinámica del capital financiero, organizado en una red interdependiente global.

Las claves para entender el surgimiento de la crisis como un punto crítico de la red financiera global son la topología propia de esa red y el carácter de los procesos que ocurren en ese tipo de redes. Sin la creciente interconectividad generada por la desregulación y expansión del capital financiero habría sido imposible que ocurriera una crisis de la magnitud de la desatada en 2008. Esa topología incluye nodos críticos, y la posibilidad de la emergencia de percolación, es decir, de la formación de conjuntos interconectados de nodos vulnerables al no pago de la deuda (o a la caída sorpresiva de los precios de los activos financieros) que generan puntos en los que el sistema "diverge" o cambia de estado (Solé, 2011).

Pero es la articulación entre la topología de la red interdependiente global y los tiempos entrelazados del capital lo que precipita la crisis. La misma metamorfosis de deuda en activos y de estos en deuda que creó la red interdependiente es lo que indujo la aparición de distintos tiempos del capital en un periodo de fuerte innovación financiera y de transformación del capitalismo global.

Por eso, no es el paso del tiempo lo que determina la evolución de las otras "cosas" (deudas de todo tipo) de que habla Ha-Joon: son las relaciones entre esas "cosas" y sus transformaciones en otras lo que genera el tiempo. El tiempo en el mundo del capital financiero es la secuencia de transformaciones de las deudas iniciales. Y ese tiempo no es el tiempo calendario ni el tiempo de los relojes. Es el tiempo de la evolución de la deuda, desde un crédito inicial y una promesa de pago, hacia la conversión final en liquidez en una estructura de red compleja.

En muchos eventos esos dos tiempos no coinciden y no es preciso que coincidan para garantizar la valorización y reproducción del capi- 
tal. Lo hacen en la explosión de la crisis: el tiempo del capital financiero coincide con el tiempo calendario cuando todos los portadores de activos desean dinero y no quieren activos ni mercancías. Hay una fecha o unas fechas para marcar el momento de la crisis, al menos para fijar su rango en términos de unidades de tiempo calendario.

E1 conjunto de las transacciones financieras generadas a partir de deuda (crédito) en múltiples puntos del sistema económico genera una compleja red de interrelaciones conformada por múltiples agentes económicos (bancos de inversión e inversionistas, capitales productivos) y los vínculos de obligaciones y activos (o promesas y deudas) que los unen. Ese sistema complejo de interrelaciones financieras tiene una dinámica propia que no es idéntica a la suma lineal de los planes individuales de inversionistas y bancos ${ }^{15}$. La crisis no aparece como resultado de la incapacidad individual para cumplir sus obligaciones o promesas de pagar en efectivo, sino de un cambio de estado del sistema en su conjunto: lo que precipita la crisis es la incapacidad colectiva de honrar las deudas en efectivo, junto a la caída vertiginosa de los precios de los activos financieros.

El evento de la crisis emerge entonces como una propiedad de un sistema de posposición de pago de las deudas que toma la forma de una red compleja de interrelaciones, que opera con una dinámica "robusta pero frágil"(Gai y Kapadia, 2010). Lo que Marx había visto como una posibilidad que surgía de la separación estructural entre el momento de la compra y de la venta, aquí aparece como un fenómeno de cambio de estado de un sistema complejo, precipitado por la conectividad alimentada por el apalancamiento creciente del sistema. Pero esa complejidad ya estaba implícita en la perspectiva compleja del tiempo sugerida por Marx en El Capital.

\section{REFERENCIAS BIBLIOGRÁFICAS}

Althusser, L., Balibar, E. et al. (2015). Reading capital. The complete edition. Londres: Verso.

Anand, K., Gai, P. et al. (2012). A network model of financial system resilience. Bank of England working paper 458.

Benjamin, W. (1999). The Arcades Project. Cambridge Mass.: Harvard University Press.

15 Analizando el valor en un contexto relacional espacio-tiempo, David Harvey muestra que "los valores relacionales cambian en tándem con los sentimientos, la confianza, las expectativas y las anticipaciones del mercado" (Harvey, 2017, p. 141). No es posible establecer el valor "objetivo" de una propiedad: su valor es relacional y cambiante y, por tanto, social. 
Bensaïd, D. (2007). Althusser et la mystère de la rencontre, [http://www. danielbensaid.org/Althusser-et-le-mystere-de-la-rencontre?lang=fr].

Bensaïd, D. (2012). Marx ha vuelto. Barcelona: Edhasa.

Chesnais, F. (2017). Las dimensiones financieras del impasse del capitalismo, [www.sinpermiso.info/textos/las-dimensiones-financieras-delimpasse-del-capitalismo].

Fama, E. (1970). Efficient capital markets: A review of theory and empirical work. Journal of Finance, 25(2), 383-417.

Gai, P. y Kapadia, S. (2010). Contagion in financial networks. Proceedings of the Royal Society A, 466(2010), 2401-2423.

Gödel, K. (1949a). A remark about the relationship between relativity theory and idealistic philosophy. En Schilpp, P. A. (ed.). Albert Einstein, philosopher-scientist. Evanston, Ill.:Northwestern University Press.

Gödel, K. (1949b). An example of a new type of cosmological solutions of Einstein's field equations of gravitation. Reviews of Modern Physics, 21(3), 447-450.

Gorton, G. (2010). Slapped by the invisible hand. The panic of 2007. Nueva York: Oxford University Press.

Ha-Joon, C. (2015). Economía para el 99\% de la población. Bogotá: Penguin Random House.

Harvey, D. (2017). Marx, capital and the madness of economic reason. Londres: Profile Books.

Hawking, S. (2008). La gran ilusión. Las grandes obras de Albert Einstein. Barcelona: Crítica.

Karatani, K. (2005). Transcritique: On Marx and Kant. Cambridge, Mass.: MIT Press.

Karatani, K. (2014). The structure of world history. From modes of production to modes of exchange. Durham: Duke University Press.

Leijonhufvud, A. (2011). Nature of an economy. CEPR Policy Insights $53,1-10$.

Marx, K. (1967). Capital, vol. III. Nueva York: International Publishers.

Marx, K. (1976). Capital, vol. I. Londres: Penguin.

Marx, K. (1978). Capital, vol. II. Londres: Penguin.

Osborne, P. (2008). Marx and the philosophy of time. Radical Philosophy, $147,15-22$.

Solé, R. V. (2011). Phase transitions. Princeton: Princeton University Press.

Stewart, J. B. (2009). Eight days. The battle to save the American financial system. The New Yorker, 21, 59-81.

Watts, D. J. (2002). A simple model of global cascades on random networks. Proceedings of the National Academy of Sciences, 99(9), 5766-5771. 